\title{
Genetic Parameters for Stillbirth in Danish Holstein Cows Using a Bayesian Threshold Model
}

\author{
M. Hansen, ${ }^{1,2,3}$ M. S. Lund, ${ }^{1}$ J. Pedersen, ${ }^{2}$ and L. G. Christensen ${ }^{3}$ \\ ${ }^{1}$ Danish Institute of Agricultural Sciences, Dept. of Animal Breeding and Genetics, \\ Research Centre Foulum, P.O. Box 50, DK-8830 Tjele, Denmark \\ ${ }^{2}$ The Danish Agricultural Advisory Centre, Udkærsvej 15, Skejby, 8200 Aarhus N, Denmark \\ ${ }^{3}$ The Royal Veterinary and Agricultural University, Dept. of Animal Science and Animal Health, \\ Grønnegårdsvej 2, DK-1870, Frederiksberg C, Denmark
}

\section{ABSTRACT}

The objective of this study was to make an inference about the direct and maternal genetic variation of stillbirth for first-calving Holstein cows and to estimate the effect of breed and heterosis for original Danish black and white and Holstein-Friesian. A Bayesian threshold model, which included correlated genetic effects of sires and maternal grandsires was used. Marginal posterior distributions of effects were obtained using Gibbs sampling. Point estimates were compared with results from a linear model using REML. Data with and without twins were analyzed and models with and without effects of breed and heterosis were fitted, but estimates of genetic parameters were almost identical. In all the analyses with threshold models, the marginal posterior mean (and standard deviation) was 0.10 (0.014) for the direct heritability, $0.13(0.015)$ for the maternal heritability, and $0.05(0.10)$ for the genetic correlation between direct and maternal effects. The stillbirth rate tended to increase with a higher proportion of HolsteinFriesian in the calf and in the dam, but no effects of breed and heterosis were significant. Joint sampling of all location parameters was found superior to univariate sampling in terms of much better mixing properties of the fixed effects. Based on the results showing genetic variation for stillbirth at first calving, both the direct and the maternal effect could be included in the breeding program.

(Key words: stillbirth, Holstein-Friesian, genetic parameter, threshold model)

\begin{abstract}
Abbreviation key: $\mathbf{C G}=$ contemporary group, $\mathbf{E C P}=$ extreme category problem, $\mathbf{H F}=$ Holstein-Friesian, MGS = maternal grandsire, $\mathbf{O D B W}=$ original Danish black and white, S-MGS = sire-maternal grandsire.
\end{abstract}

Received January 26, 2003.

Accepted July 14, 2003.

Corresponding author: M. Hansen; e-mail: mxh@lr.dk.

\section{INTRODUCTION}

During the last 2 decades in Denmark the incidence of stillbirths for Holstein cattle has increased (Nielsen et al., 2002). The same trend has been observed in Sweden (Steinbock et al., 2003) and in the United States (Meyer et al., 2001a). Stillbirths in dairy cattle herds reduce the potential number of replacement heifers and reduce the revenue from bulls for fattening, but it is also an ethical problem related to animal welfare. Stillbirth has been shown to be heritable due to additive genetic effects in the calf (the direct effect) and to additive genetic effects in the dam (the maternal effect) (Philipsson, 1976; Weller et al., 1988, Luo et al., 1999). This means that stillbirth is a potential trait to include in a breeding program, and, therefore, exact knowledge of genetic parameters for this trait is needed.

Many studies of genetic parameters for stillbirth have applied linear models and thereby ignored the categorical nature of stillbirth data (e.g., Niskanen and Juga, 1998; Luo et al., 1999; Harbers et al., 2000; Meyer et al., 2001b). From these studies, the direct and maternal heritabilities ranged from 0.01 to 0.06 . It is not straightforward to compare these heritabilities, because they will depend on the frequency of stillbirth in the population analyzed. To compare heritabilities obtained with linear models, they can be transformed to a scale of underlying liabilities using the formula from Robertson in Dempster and Lerner (1950). However, this transformation has been shown to give biased results when the frequency is low (Van Vleck, 1972). It is desirable to analyze stillbirth data with a model that directly takes account of the categorical nature of these data, such as the threshold model (Wright, 1934). The threshold model assumes an unobservable underlying continuous variable (liability), with one or more thresholds deciding the observed categorical outcome. An empirical Bayesian method to draw inference about variance components in a threshold model was proposed by Harville and Mee (1983). This method relies on asymptotic properties and has been found to give biased inferences (Hoeschele and Gianola, 1989; Hoeschele and Tier, 
Table 1. Reasons for omitting a record, number of records omitted, and numbers left in the stepwise editing procedure.

\begin{tabular}{lcc}
\hline Reason for omitting a record & No. omitted & No. left \\
\hline & & 391186 \\
Unknown sire & 56254 & 334932 \\
Sire was a private owned bull & 47044 & 287888 \\
Unknown maternal grandsire & 17585 & 270303 \\
Maternal grandsire was a private owned bull & 9826 & 260477 \\
Twin 1 & 3818 & 256659 \\
Dam is not biological dam due to embryo transfer & 6 & 256653 \\
Calving age above 35 mo & 6916 & 249737 \\
Calving age below 22 mo & 390 & 249347 \\
Herd with 5 or fewer calves with unproven sires & 33007 & 216340 \\
Herd with 10 or fewer calves per year & 23599 & 192741 \\
Sire with 10 or fewer calves & 2698 & 190043 \\
Maternal grandsire with 10 or fewer calves & 3425 & 186618 \\
Last 3 edits repeated until they all are fulfilled & 2167 & 184451 \\
Contemporary group with 5 or fewer records & 310 & 184141 \\
\hline
\end{tabular}

${ }^{1}$ Records of twins were not omitted for one of the analyses.

1995). Sorensen et al. (1995) showed that exact and full Bayesian inference about the marginal (and joint) posterior distributions of all parameters in a threshold model could be accomplished by use of Gibbs sampling (Geman and Geman, 1984). Sorensen et al. (1995) implemented the Gibbs sampler with augmentation of liabilities as described in Albert and Chip (1993). Only a few studies of stillbirth have used this approach. Luo (1999) used this approach for a threshold sire-maternal grandsire (S-MGS) model [a model containing correlated genetic effects of sires and maternal grandsires (MGS)] but did not succeed with the analysis as the sire variance was trapped at zero. Steinbock et al. (2003) used a similar approach and found marginal posterior means of direct and maternal heritabilities on 0.12 and 0.08 for stillbirth in first calving Swedish Holstein cows.

The proportion of genes in the Danish Holstein population descending from the original Danish black and white population in the 1960s (ODBW) has decreased since an upgrading with Holstein-Friesian (HF) began in the 1970s. In Danish Holstein calves today, the proportion of genes originating from $\mathrm{HF}$ is higher than $90 \%$. During this upgrading period, the stillbirth rate at first calving has increased in the Danish Holstein breed. A similar pattern has been observed in the Swedish Holstein breed (Steinbock et al., 2003). Therefore, it is possible that genes descending from HF have influenced the stillbirth rate. The current model used for the official Danish breeding value evaluation of stillbirth includes covariates of the proportion of ODBW and $\mathrm{HF}$ and the heterozygosity between these 2 breeds for both the calf and the dam (Danish Cattle Federation, 2002) (the way heterozygosity is defined will be explained later). Results from this evaluation indicated an unfavorable breed effect of $\mathrm{HF}$ and a small favorable effect of heterozygosity for both the direct and the maternal effect (Pedersen, 2002, personal communication). But this model is a linear model and does not account for the categorical nature of stillbirth. Inference about effects of breed and heterozygosity can be different in a threshold model.

The objectives with this study were: 1 ) to estimate the direct and maternal heritability for stillbirth at first calving in the Danish Holstein population using a Bayesian threshold model with Gibbs sampling; 2) to compare these heritabilities with heritabilities from a linear model transformed to the liability scale with the formula by Robertson in Dempster and Lerner (1950); 3 ) to test the hypotheses that the additive genetic effect of $\mathrm{HF}$ relative to the additive genetic effect of ODBW is unfavorable, and the effect of heterozygosity between $\mathrm{HF}$ and $\mathrm{ODBW}$ is favorable.

\section{MATERIALS AND METHODS}

\section{Data}

Stillbirth was defined as a calf born dead or dying within $24 \mathrm{~h}$ after birth, as recommended by Philipsson et al. (1979). Data about stillbirths have been routinely collected from all calvings on Danish dairy farms since 1986 and stored in a national database for cattle (Bundgaard and Hoej, 2000). To get a data set with a size that made computations feasible, only records from the southwestern part of Denmark were extracted. This process resulted in 391,186 records of calves born from first calving Holstein cows in the period from January 1988 to May 2002.

Editing. The editing procedure is summarized in Table 1 . The main editing rules were that the sire and the MGS of the calf had to be a known AI-bull with 10 
Table 2. Percentage of all records and percentage of stillbirth of calves depending on the status of the sire and the status of the MGS for the calf.

\begin{tabular}{lcc}
\hline & \% All & \\
& records & $\%$ stillbirth \\
\hline Danish unproven sire & 14.0 & 12.2 \\
Danish proven sire & 76.5 & 9.9 \\
Foreign sire & 9.5 & 11.4 \\
Danish unproven MGS & 28.0 & 11.4 \\
Danish proven MGS & 63.1 & 9.7 \\
Foreign MGS & 8.9 & 12.1 \\
All records & 100.0 & 10.4 \\
\hline
\end{tabular}

records as sire or as MGS. Twins were omitted for most analyses, but they were kept for one analysis. Records were clustered in subclasses of contemporary groups (CG) using the method described by Schmitz et al. (1991) with a maximum interval on $2 \mathrm{yr}$ and a minimum desired size of clusters on 20. Due to this method, a few CG subclasses were small, and records in CG subclasses with 5 or fewer records were omitted.

Bulls. Usually, only proven AI-bulls with superior predicted breeding values for the direct effect of stillbirth and calving ease have been chosen for mating with heifers. However, in Denmark it has been recommended to use some unproven AI-bulls on heifers to achieve a reliable prediction of breeding values for the direct effect of stillbirth and calving ease at first calving. In the final data set, $14 \%$ of the calves had an unproven sire and $28 \%$ of the dams had an unproven sire (Table 2). A sire or a MGS was regarded as unproven when it was less than $5 \mathrm{yr}$ old when the calf was born or when the dam was born, respectively. The final data set included 983 bulls as sires and 1055 bulls as MGS (Table 3 ). In total, 823 bulls had records both as a sire and as a MGS, and 1205 different bulls were included as a sire or as a MGS. Pedigree for these bulls was traced on both their sire and their dam paths as far back as parents were known. This resulted in an additive relationship matrix (A) consisting of 4445 animals. Most of the bulls with records were Danish AI-bulls, but some were foreign AI-bulls. Danish AI-bulls were regarded as unproven if they had progeny included that were born less than $5 \mathrm{yr}$ after the sire, or else they were regarded as proven. In total, 100 sires and 118 MGS were regarded

Table 3. Number of Danish unproven bulls, Danish proven bulls, foreign bulls, and bulls in total as sires and maternal grandsires (MGS).

\begin{tabular}{llllr}
\hline & $\begin{array}{l}\text { Danish } \\
\text { unproven }\end{array}$ & $\begin{array}{l}\text { Danish } \\
\text { proven }\end{array}$ & Foreign & Total \\
\hline Sires & 738 & 101 & 144 & 983 \\
MGS & 791 & 118 & 146 & 1055 \\
\hline
\end{tabular}

as Danish proven AI-bulls. These bulls could have been selected for fewer stillbirths based on data not included in the analyses. Selection of Danish proven AI-bulls for mating with heifers seemed to exist as $9.9 \%$ of their calves were stillborn compared with $12.2 \%$ for unproven sires. Also, calves with a proven Danish MGS had a lower incidence of stillbirth than calves from an unproven MGS. When some sires used for estimation of genetic parameters were selected for the trait of interest, Van Vleck (1985) suggested treating them as nongenetic effects to avoid selection bias. A disadvantage of this approach is that the genetic and environmental effects become less connected. This procedure would provide greater uncertainty for the genetic parameters and was not done in this study.

Breed and heterosis. The proportion of genes in the calf and in the dam originating from the ODBW and the HF population could be traced from pedigree information due to full identification of dams, sires, and MGS for all records. If an ancestor was unknown, this ancestor was given average breed proportions for this period of time. In the final data set, the average proportion of $\mathrm{HF}$ genes for calves born in 1988 was 66 and $55 \%$ for their dams. In 2002, the proportion of HF genes had increased to $94 \%$ for the calves and $90 \%$ for their dams. Heterosis was assumed to arise from effects of dominance only and therefore proportional to the degree of heterozygosity. The degree of heterozygosity (h) was obtained for each individual (calf and dam) as: $\mathrm{h}=$ psire $_{\mathrm{HF}} *$ pdam $_{\mathrm{ODBW}}+$ psire $_{\mathrm{ODBW}} *$ pdam $_{\mathrm{HF}}$, where psire and pdam were the proportion of the breed (HF or ODBW) for the sire and for the dam of the individual.

\section{Statistical Procedures}

Threshold model. We assumed that the observed outcome $(y i)$ for the $i$ 'th calf $(i=1,2, \ldots, n)$ followed a Bernoulli distribution (stillborn $=1$, alive $=0$ ), where $n$ is the total number of records included. We assumed that each calf had an unknown liability for stillbirth $\left(U_{i}\right)$. All calves with liabilities exceeding the threshold $(t)$ were observed as stillborn. The model for the liabilities was in matrix notation:

$$
\mathrm{U}=\mathbf{X b}+\mathbf{W h}+\mathbf{Z}_{1} \mathbf{s}+\mathrm{Z}_{2} \mathbf{m g s}+\mathbf{e},
$$

where $\mathbf{U}$ was a vector of liabilities, $\mathbf{b}$ was a vector of nongenetic effects plus effects of breed and heterosis, h was a vector of CG effects, $\mathbf{s}$ was a vector of sire effects, mgs was a vector of MGS effects, and e was a vector of residuals. $\mathbf{X}, \mathbf{W}, \mathbf{Z}_{1}$, and $\mathbf{Z}_{\mathbf{2}}$ were incidence matrices relating the effects to liabilities.

Three different analyses were carried out with the threshold model: TM, TM-TWIN, and TM-HET. Effects 
of breed and heterosis were only included in TM-HET and records of twins were only included in TM-TWIN. The vector $\mathbf{b}$ contained cross-classified effects of: sex of calf (male, female), month of calving $(1,2, \ldots, 12)$, calving age in months $(22,23, \ldots, 35)$, year of calving $(1988, \ldots, 2002)$, twin (single, twin) (only included for TM-TWIN). In TM-HET, b also contained the coefficients of the regressions $b_{\mathrm{HF}-\text { calf }}$ and $b_{\mathrm{HF}-\mathrm{dam}}$ for the proportion of $\mathrm{HF}$ in the calf and in the dam and $b_{\text {Het-calf }}$ and $b_{\text {Het-dam }}$ for the degree of heterozygosity between $\mathrm{HF}$ and ODBW in the calf and in the dam.

It was assumed that the liabilities conditional on all effects were independent and normally distributed:

$$
\mathbf{U} \mid \mathbf{b}, \mathbf{h}, \mathbf{s}, \mathbf{m g s} \sim \mathbf{N}_{\mathrm{n}}\left(\mathbf{X b}+\mathbf{W h}+\mathbf{Z}_{1} \mathbf{s}+\mathbf{Z}_{2} \mathbf{m g s}, \mathbf{I} \sigma_{\mathrm{e}}^{2}\right) .
$$

To obtain identifiable parameters, the residual variance $\left(\sigma_{\mathrm{e}}^{2}\right)$ was set to one, and the threshold was set to zero (Sorensen et al., 1995). The conditional probability that record $i\left(y_{i}\right)$ falls into the category "stillborn" given $\mathbf{b}, \mathbf{h}, \mathbf{s}$, and $\mathbf{m g s}$ was:

$$
\begin{aligned}
\mathrm{P}\left(y_{i}=\right. & 1 \mid \mathbf{b}, \mathbf{h}, \mathbf{s}, \mathbf{m g s})=\mathrm{P}\left(U_{i}>\mathrm{t} \mid \mathbf{b}, \mathbf{h}, \mathbf{s}, \mathbf{m g s}\right) \\
=1 & -\boldsymbol{\Phi}\left(0-\left(\mathbf{x}_{\mathrm{i}} \mathbf{b}+\mathbf{w}_{\mathrm{i}} \mathbf{h}+\mathbf{z}_{\mathrm{li}} \mathbf{s}+\mathbf{z}_{2 \mathrm{i}} \mathbf{m g s}\right)\right) \\
& =\boldsymbol{\Phi}\left(\mathbf{x}_{\mathrm{i}} \mathbf{b}+\mathbf{w}_{\mathrm{i}} \mathbf{h}+\mathbf{z}_{\mathrm{li}} \mathbf{s}+\mathbf{z}_{2 \mathrm{i}} \mathbf{m g s}\right),
\end{aligned}
$$

where $\Phi$ is the cumulative distribution function of a standard normal distribution, and $\mathbf{x}_{\mathrm{i}}, \mathbf{w}_{\mathrm{i}}, \mathbf{z}_{\mathrm{li}}$, and $\mathbf{z}_{2 \mathrm{i}}$ are row $i$ of each of the respective incidence matrices.

Priors for the location parameters were:

$$
\begin{gathered}
\mathrm{p}(\mathbf{b})\left(\mathrm{N}\left(\mathbf{0}, 10^{6}\right),\right. \\
\mathrm{p}\left(\mathbf{h} \mid \sigma_{\mathrm{CG}}^{2}\right)\left(\mathrm{N}\left(\mathbf{0}, \mathbf{I} \sigma_{\mathrm{CG}}^{2}\right),\right. \text { and } \\
\mathrm{p}\left(\left[\begin{array}{c}
\mathbf{s} \\
\mathbf{m g s}
\end{array}\right] \mid \mathbf{G}_{0}, \mathbf{A}\right) \sim \mathrm{N}\left(\mathbf{0}, \mathbf{G}_{0} \otimes \mathbf{A}\right),
\end{gathered}
$$

where $\mathbf{G}_{0}=\left[\begin{array}{cc}\sigma_{\text {sire }}^{2} & \sigma_{\text {sire,mgs }} \\ \sigma_{\text {sire,mgs }} & \sigma_{\text {mgs }}^{2}\end{array}\right]$ and $\mathbf{A}$ is the additive genetic relationship matrix consisting of bulls, which are sires or MGS of calves, and all their known ancestors.

Conjugate proper prior distributions were used for the dispersion parameters. The prior for $\sigma_{\mathrm{CG}}^{2}$ was an inverted chi-square distribution with 4 degrees of freedom and with an expectation $=0.11511$. The prior for $\mathbf{G}_{0}$ was a two-dimensional inverted Wishart distribution with 4 degrees of freedom and with an expectation of the (co)variance matrix $=\left[\begin{array}{ll}0.02878 & 0.01439 \\ 0.01439 & 0.03597\end{array}\right]$. The expectations of these priors were chosen such that, after transformation to the derived variables (described in the following), they were equivalent to a direct heritability of 0.10 , a maternal heritability of 0.10 , a correlation between direct and maternal effects of zero, and a ratio of the variance of $C G$ effects to the phenotypic variance of 0.10 . These prior expectations were based on estimates from Luo (1999) and Steinbock et al. (2003).

To obtain marginal posterior distributions of derived variables, they were generated from each saved sample of dispersion parameters. Direct and maternal genetic (co)variances were generated following derivations from Willham (1972):

$$
\begin{gathered}
\sigma_{\mathrm{D}}^{2}=4 \sigma_{\mathrm{S}}^{2}, \\
\sigma_{\mathrm{D}, \mathrm{M}}=4 \sigma_{\mathrm{S}, \mathrm{MGS}}-2 \sigma_{\mathrm{S}}^{2}, \text { and } \\
\sigma_{\mathrm{M}}^{2}=4 \sigma_{\mathrm{MGS}}^{2}-4 \sigma_{\mathrm{S}, \mathrm{MGS}}+\sigma_{\mathrm{S}}^{2}
\end{gathered}
$$

The genetic correlation between direct and maternal effects was generated as:

$$
\mathrm{r}_{\mathrm{D}, \mathrm{M}}=\frac{\sigma_{\mathrm{D}, \mathrm{M}}}{\sqrt{\sigma_{\mathrm{D}}^{2} \sigma_{\mathrm{M}}^{2}}}
$$

The expectation of the phenotypic variance for this model was:

$$
\sigma_{\mathrm{P}}^{2}=\sigma_{\mathrm{S}}^{2}+\sigma_{\mathrm{MGS}}^{2}+\sigma_{\mathrm{CG}}^{2}+\mathrm{a}_{\mathrm{S}, \mathrm{MGS}} 2 \sigma_{\mathrm{S}, \mathrm{MGS}}+\sigma_{\mathrm{E}}^{2},
$$

where $a_{S, M G S}$ was the genetic relationship between the sire and the MGS. When we calculated the phenotypic variance we assumed that the sire and the MGS were unrelated. Therefore, the term $\mathrm{a}_{\mathrm{S}, \mathrm{MGS}} 2 \sigma_{\mathrm{S}, \mathrm{MGS}}$ dropped out. Using this phenotypic variance, the direct and the maternal heritabilities were generated as: $\mathrm{h}_{\mathrm{D}}^{2}=\frac{\sigma_{\mathrm{D}}^{2}}{\sigma_{\mathrm{P}}^{2}}$ and $\mathrm{h}_{\mathrm{M}}^{2}=\frac{\sigma_{\mathrm{M}}^{2}}{\sigma_{\mathrm{P}}^{2}}$, and a CG ratio was generated as:

$$
\operatorname{cg}^{2}=\frac{\sigma_{\mathrm{CG}}^{2}}{\sigma_{\mathrm{P}}^{2}}
$$

Draws from the posterior distribution of the parameters in the model were obtained by Gibbs sampling (Geman and Geman, 1984). The Gibbs sampling approach was carried out by software developed by Korsgaard et al. (2003), which is implemented in the DMUpackage (Madsen and Jensen, 2000). In this software two different methods to sample location parameters are implemented: univariate and joint sampling. In the joint sampling, all location parameters are sampled simultaneously based on the method by García-Cortés and Sorensen (1996), which do not need inversion of 
the coefficient matrix of the mixed model equations. Joint sampling can often reduce the lag correlation between subsequent samples (Liu et al., 1994), but the computing time for one round in the Gibbs sampler is 2 to 3 times longer than univariate sampling (for the current analyses). For each analysis, 2 chains (A and B) consisting of 60,000 to 86,000 rounds were carried out using univariate sampling of location parameters. Additionally, two chains (C and D) each on 21,000 rounds were carried out for TM-HET where joint sampling of location parameters was used.

The length of the burn-in period was assessed by visual examination of trace plots of all effects in $\mathbf{b}$, all (co)variance components, and all derived variables. In the chains with univariate sampling of location parameters, samples from the first 10,000 rounds were discarded as burn-in period for TM-TWIN and TM, but in TM-HET 40000 rounds were discarded. In the chains with joint sampling, only 2000 rounds were discarded. The samples after burn-in were divided into 20 sequential batches. The means of the batches should converge to independent and identically distributed normal random variables. This was assessed by calculating lag correlations between batch means and performing oneway analyses of variance of the batch means. Effective sample size was calculated for each parameter by dividing the variance within batch with the variance between batches (e.g., Sorensen and Gianola, 2002).

Summary statistics of nongenetic effects were given as the predicted probabilities of stillbirth for a calf conditional on marginal posterior means of all nongenetic effects. The predicted probability of stillbirth in TMTWIN for, e.g., a single born male calf with a 22-mo-old dam born in January 2002 was the sum of the marginal posterior means of these nongenetic effects evaluated in the cumulative distribution function of a standard normal distribution.

Linear model. The linear models included the same effects as the threshold model, but the response variable was the actual observation ( 1 or 0 ). Estimates of (co)variance components were obtained with AI-REML (Jensen et al., 1997) using the DMU-package (Madsen and Jensen, 2000). Two analyses were carried out: LM and LM-HET, where LM-HET included effects of breed and heterosis.

\section{RESULTS}

\section{Heritabilities}

The marginal posterior distributions of direct and maternal heritabilities (Figure 1) were both relatively symmetric. For all the analysis, the two chains (A and B) gave very similar inference (Table 4). The marginal posterior mean of the direct heritability from TM-TWIN

\section{1}

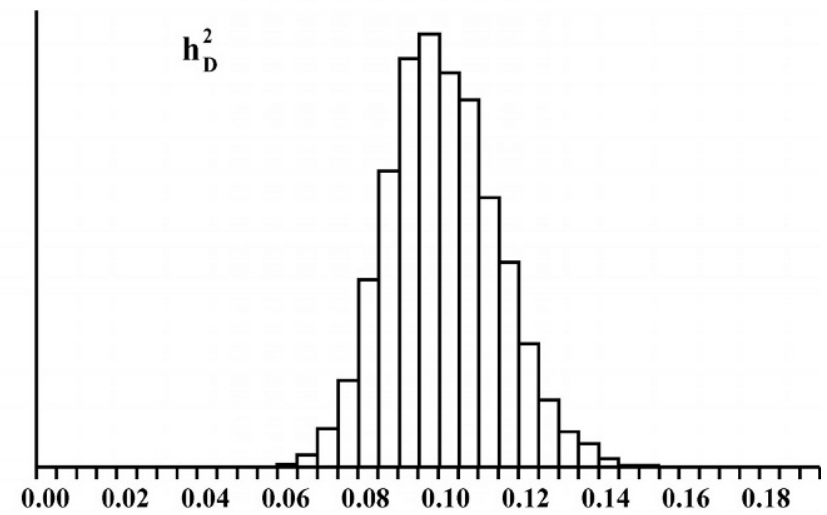

2

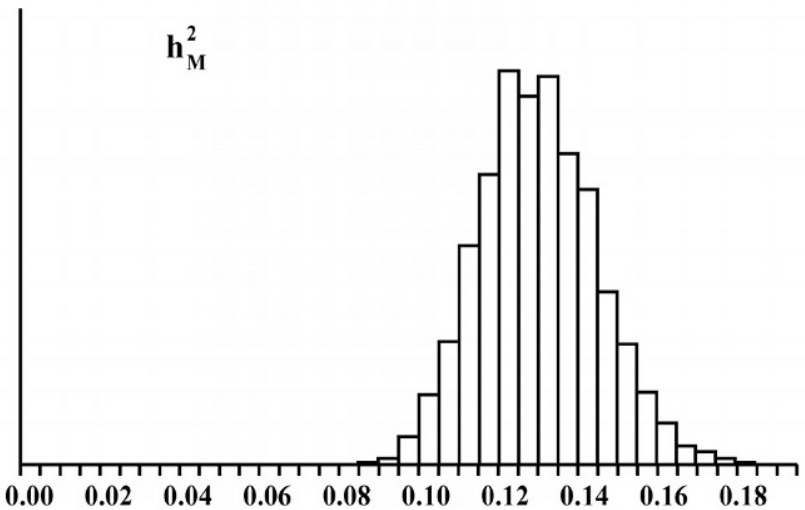

3

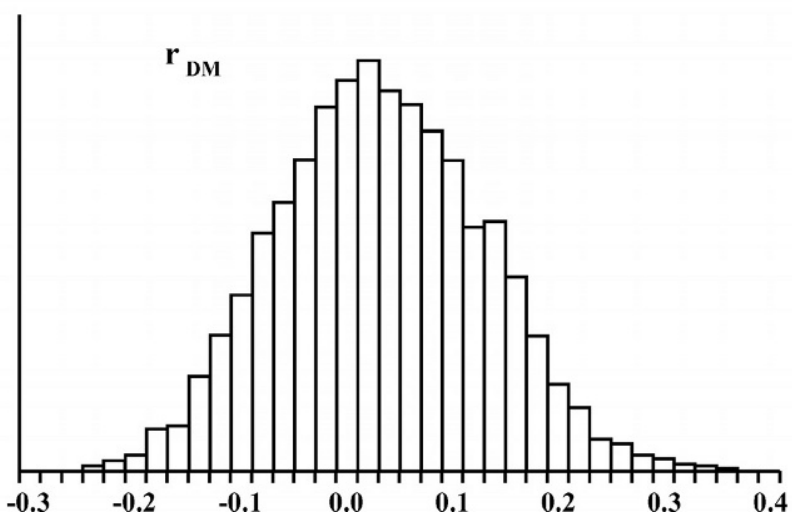

Figure 1. Histogram of samples from TM-TWIN showing the marginal posterior distribution of the direct heritability $\left(\mathrm{h}_{\mathrm{D}}^{2}\right)$, the maternal heritability $\left(\mathrm{h}_{\mathrm{M}}^{2}\right)$, and the genetic correlation between direct and maternal effects $\left(r_{\mathrm{DM}}\right)$.

was 0.10 and the standard deviation was 0.014 . The marginal posterior mean of the maternal heritability from the same analysis was 0.13 , and the standard deviation was 0.015 . Only small differences in these 
Table 4. Effective sample size (ESS) and summary statistics of marginal posterior distributions of phenotypic variation $\left(\sigma_{\mathrm{P}}^{2}\right)$, CG ratio $\left(\mathrm{cg}^{2}\right)$, direct and maternal heritability $\left(\mathrm{h}_{\mathrm{D}}^{2}\right.$ and $\left.\mathrm{h}_{\mathrm{M}}^{2}\right)$, and genetic correlation between direct and maternal effects $\left(\mathrm{r}_{\mathrm{D}, \mathrm{M}}\right)$ from chain $\mathrm{A}$ and B in TM-TWIN, TM, and chain A, B, C, and D in TMHET.

\begin{tabular}{|c|c|c|c|c|c|c|}
\hline Analysis & & $\sigma_{\mathrm{P}}^{2}$ & $\mathrm{cg}^{2}$ & $\mathrm{~h}_{\mathrm{D}}^{2}$ & $\mathrm{~h}_{\mathrm{M}}^{2}$ & $\mathrm{r}_{\mathrm{D}, \mathrm{M}}$ \\
\hline \multirow{5}{*}{ TM-TWIN } & ESS A & 1093 & 1466 & 986 & 559 & 773 \\
\hline & ESS B & 589 & 1461 & 792 & 668 & 649 \\
\hline & Mean A & 1.098 & 0.025 & 0.099 & 0.128 & 0.032 \\
\hline & Mean B & 1.098 & 0.025 & 0.099 & 0.126 & 0.041 \\
\hline & $\mathrm{SD} A$ & 0.006 & 0.002 & 0.014 & 0.015 & 0.098 \\
\hline TIVI & ESS A & 313 & 702 & 257 & 299 & 273 \\
\hline & ESS B & 680 & 925 & 670 & 350 & 409 \\
\hline & Mean A & 1.095 & 0.024 & 0.097 & 0.126 & 0.061 \\
\hline & Mean B & 1.095 & 0.023 & 0.097 & 0.127 & 0.054 \\
\hline & $\mathrm{SD} \mathrm{A}$ & 0.006 & 0.002 & 0.014 & 0.015 & 0.107 \\
\hline \multicolumn{7}{|l|}{ TM-HET $^{1}$} \\
\hline & ESS B & 309 & 638 & 197 & 257 & 230 \\
\hline & Mean A & 1.096 & 0.024 & 0.098 & 0.129 & 0.044 \\
\hline & Mean B & 1.095 & 0.024 & 0.098 & 0.128 & 0.041 \\
\hline & SD A & 0.006 & 0.002 & 0.015 & 0.015 & 0.105 \\
\hline TM-HE'T & ESS C & 363 & 609 & 168 & 263 & 128 \\
\hline & ESS D & 300 & 301 & 249 & 187 & 152 \\
\hline & Mean C & 1.096 & 0.024 & 0.099 & 0.128 & 0.054 \\
\hline & Mean D & 1.096 & 0.023 & 0.099 & 0.127 & 0.051 \\
\hline & SD C & 0.007 & 0.002 & 0.014 & 0.014 & 0.099 \\
\hline
\end{tabular}

${ }^{1}$ Univariate sampling of location parameters.

${ }^{2}$ Joint sampling of location parameters.

estimates were observed when twins were discarded and when effects of breed and heterosis were not included in the model (Table 4).

The linear models (LM and LM-HET) resulted in estimates of heritabilities for direct effects on 0.05 and for maternal effects on 0.06 with a standard error on 0.006 for both estimates (Table 5). The transformation of these heritabilities from LM to the liability scale gave a heritability of 0.15 for direct effects and 0.17 for maternal effects.

\section{Genetic Correlation}

The marginal posterior distribution of the correlation between direct and maternal effects was symmetric
(Figure 1), with means from 0.03 to 0.06 and standard deviations from 0.10 to 0.11 (Table 4). The estimates of the correlation from the linear models were both -0.04 , with standard errors on 0.09 (Table 5).

\section{Breed and Heterosis}

The marginal posterior distributions for effects of breed and heterosis were very inaccurate for the two chains, which used univariate sampling of location parameters (A and $\mathrm{B})$. In contrast, the two chains with joint sampling $(\mathrm{C}$ and $\mathrm{D})$ gave much more accurate inferences. For a computing time on 20 to $25 \mathrm{~d}$, only 20 to 43 effective samples were obtained with the univari-

Table 5. Estimates of phenotypic variation $\left(\sigma_{\mathrm{P}}^{2}\right)$, CG ratio $\left(\mathrm{cg}^{2}\right)$, direct and maternal heritability $\left(\mathrm{h}_{\mathrm{D}}^{2}\right.$ and $\mathrm{h}_{\mathrm{M}}^{2}$ ), and genetic correlation between direct and maternal effects $\left(\mathrm{r}_{\mathrm{D}, \mathrm{M}}\right)$ from the linear models (LM and LMHET) on the observed scale (Obs) with standard errors (in brackets) and transformed to the liability scale (Lia).

\begin{tabular}{|c|c|c|c|c|}
\hline & \multicolumn{2}{|c|}{ LM } & \multicolumn{2}{|c|}{ LM-HET } \\
\hline & Obs & Lia & Obs & Lia \\
\hline$\sigma_{\mathrm{P}}^{2}$ & $0.091_{(0.003)}$ & .1 & $0.091_{(0.003)}$ & $\ldots^{1}$ \\
\hline $\operatorname{cg}^{2}$ & 0.008 & 0.023 & $0.008_{(0.001)}$ & 0.023 \\
\hline $\mathrm{h}_{\mathrm{D}}^{2}$ & $0.050_{(0.006)}$ & 0.146 & $0.051_{(0.006)}$ & 0.148 \\
\hline $\mathrm{h}_{\mathrm{M}}^{2}$ & $0.058(0.006)$ & 0.168 & $0.058(0.006)$ & 0.169 \\
\hline $\mathrm{r}_{\mathrm{D}, \mathrm{M}}$ & $-0.035(0.089)$ & & $-0.040(0.088)$ & \\
\hline
\end{tabular}

${ }^{1}$ No transformation was carried out. 

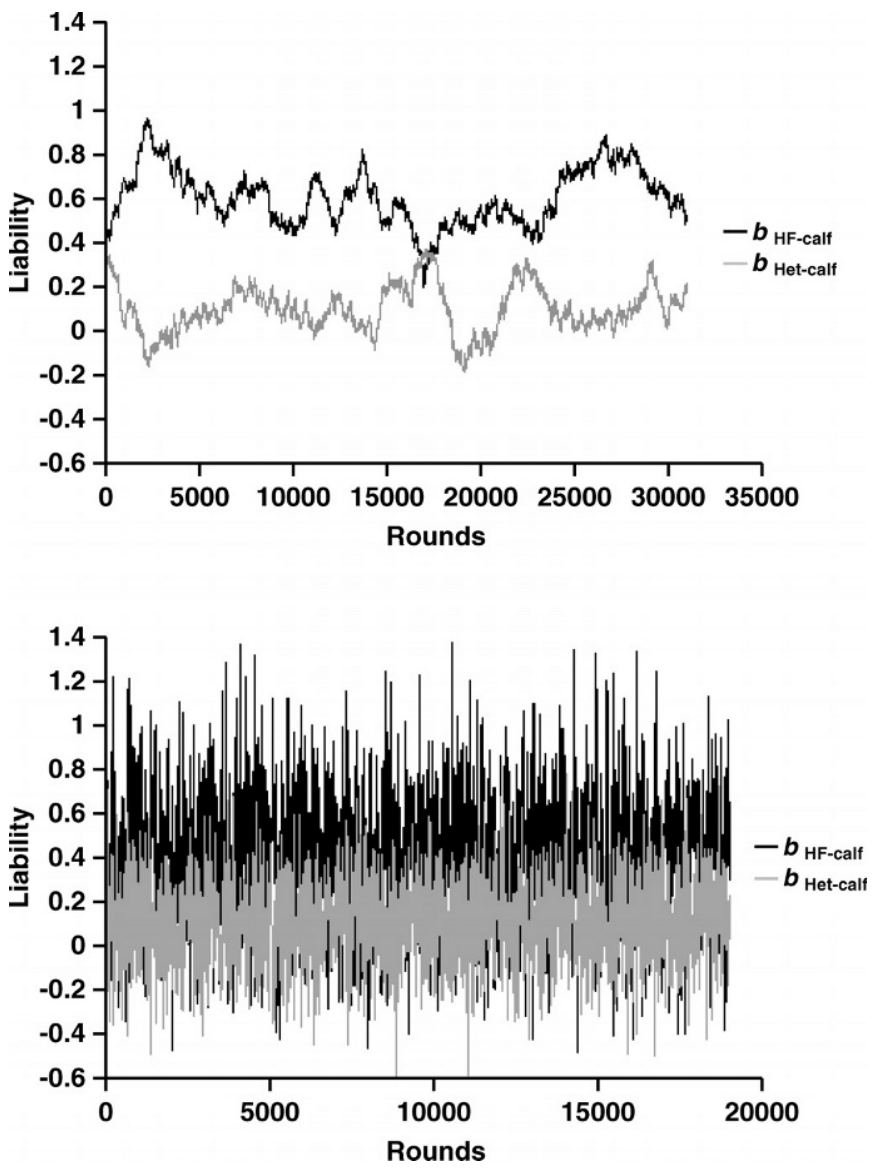

Figure 2. Traceplot of posterior samples of $b_{\text {HF-calf }}$ and $b_{\text {Het-calf }}$ after burn-in for chain A (upper graph) with univariate sampling of location parameters and for chain $\mathrm{C}$ (lower graph) with joint sampling of location parameters.

ate sampling, but more than 2750 were obtained with the joint sampling. The reason for these differences was a considerable difference in mixing properties for the methods as illustrated in Figure 2. With univariate sampling, the lag 300 correlations for all effects of breed and heterosis were from 0.45 to 0.95 , but with the joint sampling the lag 10 correlation was below 0.05 .

The analyses with joint sampling of location parameters showed that the effect of HF in the calf and in the dam was unfavorable. However, the effects of breed and heterosis were not considered significant, as zero was included in the 95\% central posterior interval (Table 6 and Figure 3). The largest and most significant effect was the effect of $\mathrm{HF}$ in the dam, which had a marginal posterior mean of 0.50 . This effect can be interpreted as the difference between a dam with $100 \% \mathrm{HF}$ genes compared with a dam with $100 \%$ ODBW genes. When the posterior mean of this effect was transformed to the observable scale, the probability of stillbirth was $13.9 \%$ for dams with $100 \%$ HF genes, given that the stillbirth rate was $7 \%$ for dams with $100 \%$ ODBW genes. The estimates of breed and heterosis from the linear model were generally in accordance with the effects from the threshold model (Table 6).

\section{Nongenetic Effects}

The marginal posterior distribution of the CG ratio was symmetric and had a small standard deviation. The posterior mean (standard deviation) was 0.025 (0.002) in TM-TWIN and $0.024(0.002)$ in the other threshold models. Calves from dams with a low calving age had a high probability of stillbirth (Figure 4). For a calf born in January 2002 from a dam with a calving age at $22 \mathrm{mo}$, the probability was 0.29 for a male and 0.21 for a female. For dams with a calving age of 28 mo or older, the probability was around 0.15 for male calves and 0.10 for female calves. The probability of stillbirth for calves born in July, August, and September was about 0.010 to 0.015 lower than for calves born in autumn and early winter (results not shown).

\section{DISCUSSION}

\section{Heritabilities}

The estimated heritabilities were, in general, low, with a maternal heritability slightly higher than the direct heritability. Heritabilities obtained by Weller et al. (1988) and Steinbock et al. (2003) are in accordance with this study, but Luo (1999) found a direct heritability on 0.016 and a maternal heritability on 0.027 . Luo (1999) believed that low data quality was the reason for these very low heritabilities. From studies using linear models, Luo et al. (1999) found heritabilities very similar to this study. Niskanen and Juga (1998), Harbers et al. (2000), and Meyer et al. (2001b) found lower heritabilities. However, all these studies fitted gestation length as a fixed effect in the model, so they are not directly comparable with this study.

The transformation of heritabilities obtained from linear models to the liability scale with the formula by Robertson in Dempster and Lerner (1950) gave an upward bias. The same upward bias was seen in Weller et al. (1988) and Steinbock et al. (2003). These results confirm the simulation study of Van Vleck (1972), where the transformation gave upward biased heritabilities when the frequency was low. Therefore, inference about the heritabilities on the liability scale ought to be from models accounting for the categorical nature of the data.

\section{Genetic Correlations}

The genetic association between direct and maternal effects was found to be small as the marginal posterior 
Table 6. Inference about breed and heterosis. Inference is given on the liability scale for TM-HET (chain A and B) and on the observable scale for TM-HET and LM-HET. ${ }^{3}$ Observable scale for effects from TMHET were given as increased level of probability of stillbirth relatively to a situation with a probability of stillbirth on 0.07 .

\begin{tabular}{|c|c|c|c|c|c|c|c|c|}
\hline \multirow[b]{3}{*}{ Chain } & & & \multicolumn{4}{|c|}{ Liability scale } & \multicolumn{2}{|c|}{ Observable scale } \\
\hline & \multicolumn{2}{|c|}{$\mathrm{ESS}^{1}$} & \multicolumn{2}{|c|}{$\mathrm{PM}^{2}$} & \multirow{2}{*}{$\frac{\mathrm{SD}^{3}}{\mathrm{~A}}$} & \multirow{2}{*}{$\frac{95 \% \mathrm{CPI}^{4}}{\mathrm{~A}}$} & \multirow{2}{*}{$\frac{\mathrm{PM}}{\mathrm{A}}$} & \multirow[b]{2}{*}{ LM-EST $^{5}$} \\
\hline & $\mathrm{A}$ & B & A & B & & & & \\
\hline$b_{\text {HF-calf }}$ & 2820 & 3656 & 0.39 & 0.40 & 0.32 & {$[-0.25 ; 1.00]$} & 0.069 & $0.060 \pm 0.058$ \\
\hline$b_{\text {Het-calf }}$ & 3636 & 3667 & 0.12 & 0.12 & 0.21 & {$[-0.29 ; 0.49]$} & 0.017 & $0.012_{ \pm 0.037}$ \\
\hline$b_{\text {HF-dam }}$ & 5168 & 2794 & 0.50 & 0.50 & 0.25 & {$[0.00 ; 0.97]$} & 0.093 & $0.078 \pm 0.042$ \\
\hline$b_{\text {Het-dam }}$ & 3460 & 2749 & -0.07 & -0.07 & 0.10 & {$[-0.27 ; 0.14]$} & 0.009 & $0.015_{ \pm 0.017}$ \\
\hline
\end{tabular}

${ }^{1} \mathrm{ESS}=$ Effective sample size

${ }^{2} \mathrm{PM}=$ posterior mean .

${ }^{3} \mathrm{SD}=$ standard deviation.

${ }^{4} \mathrm{CPI}=$ central posterior interval.

${ }^{5}$ LM-EST $=$ Estimates \pm standard errors obtained with the linear model using AI-REML.

mean of the correlation was 0.03 with twins included in the data and 0.06 without twins. A slightly different estimate of the genetic correlation $(-0.04)$ was obtained from the linear models. A correlation close to zero implies that selection on, e.g., the direct effect of stillbirth would not influence the maternal effect of stillbirth. The present results are in agreement with other studies using threshold models, where Luo (1999) found a correlation on 0.03 and Steinbock et al. (2003) found a correlation on -0.11 . However, it contradicts the correlation on -0.24 found by Luo et al. (1999), where a linear model was used.
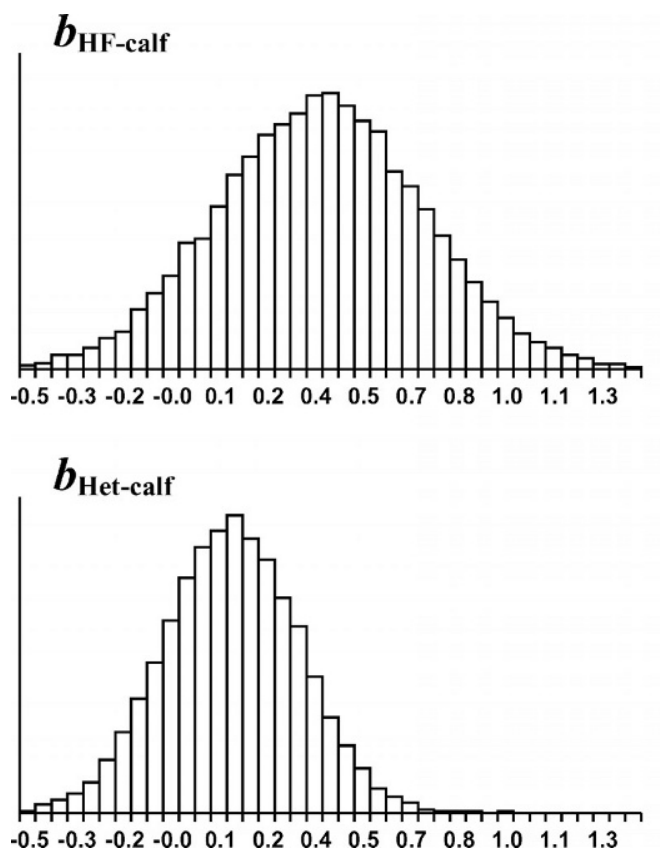

\section{Breed and Heterosis}

The indication of an unfavorable effect of $\mathrm{HF}$ on stillbirth in the Danish Holstein population (however, not significant) were confirmed in a larger study of 1.8 million calves with a threshold model assuming known (co)variances (Hansen et al. April 2003, unpublished). However, both the analyzed data sets were not well suited for inference about effects of breed and heterosis effects because only a small part of the animals had less than 50\% HF genes and because the proportion of HF genes increased through the whole period. This
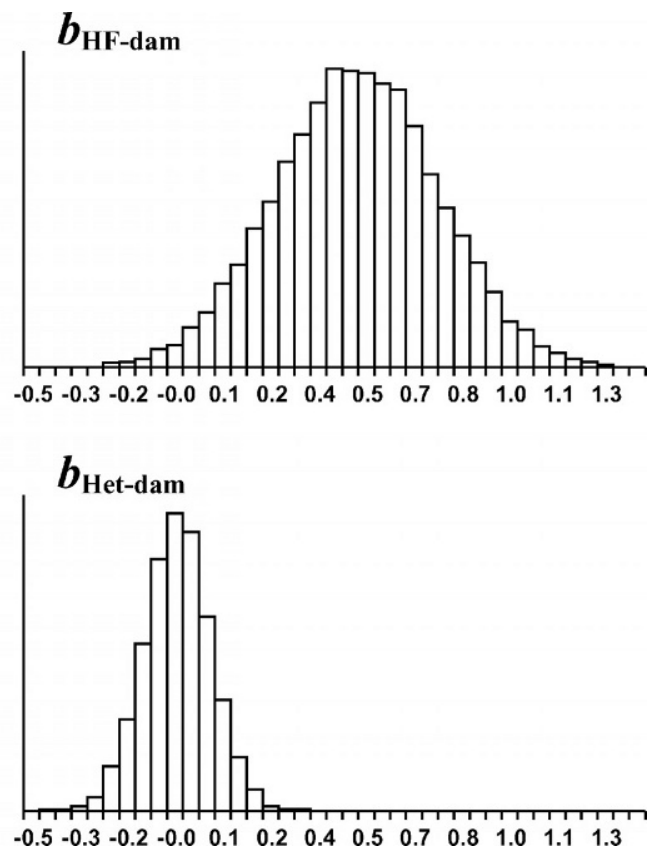

Figure 3. Histogram of posterior samples from TM-HET with joint sampling of locations parameters showing marginal posterior distributions of the effect of heterozygosity and Holstein-Friesen in the calf ( $b_{\text {Het-calf }}$ and $\left.b_{\mathrm{HF}-\text { calf }}\right)$ and in the dam $\left(b_{\mathrm{Het}-\mathrm{dam}}\right.$ and $\left.b_{\mathrm{HF}-\mathrm{dam}}\right)$. 


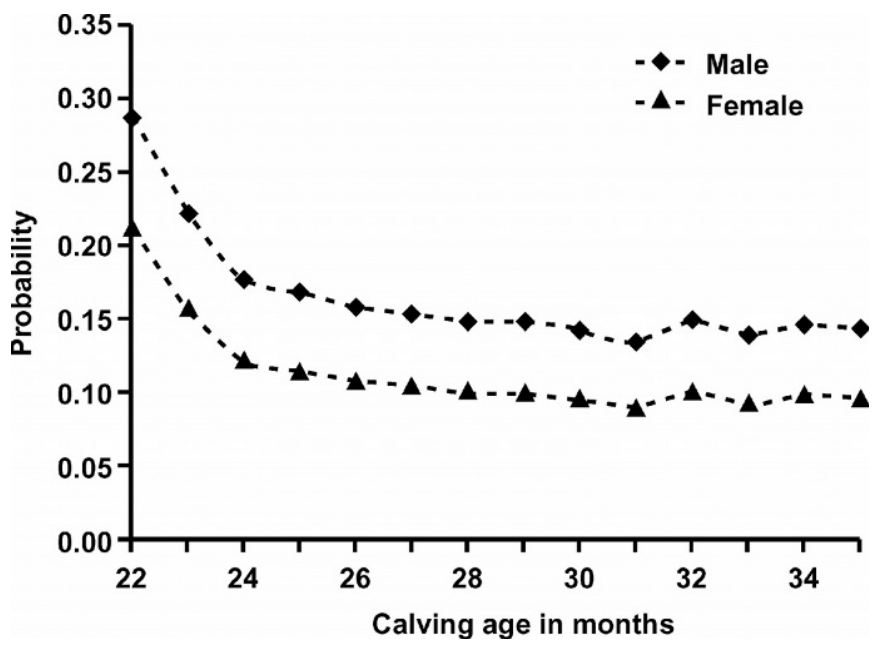

Figure 4. Probability of stillbirth depending on the calving age of the dam for male and female calves born in January 2002.

would result in some confounding between the effects of breed, heterosis, and year. A balanced data set is needed to avoid this confounding. But an important conclusion of this study was that fitting effects of breed and heterosis or not, did not change the inference about the additive genetic (co)variances.

The exact marginal posterior distributions for effects of breed and heterosis were very inaccurate with univariate sampling of location parameters due to slow mixing. The mixing problems were due to a high correlation between samples of the effects of breed, heterosis, and sex (Table 7). However, sampling location parameters jointly improved the mixing properties enormously. Therefore it seems to be very advantageous to use joint sampling when posterior samples of effects are highly correlated.

\section{Stillbirth and Calving Problems}

A major risk of stillbirth is due to a disproportion between the size of the calf and the pelvis, which will result in a complicated calving (Meijering et al., 1984). Therefore, both direct and maternal effects of calving problems are associated with stillbirth (Luo et al.,
1999), but existence of direct genetic variation of stillbirth, which is independent of calving ease, also seems plausible. First, the direct genetic correlation between stillbirth and calving ease has been found to be 0.59 (Luo et al., 1999). Secondly, the direct heritability of stillbirth was 0.022 when calving ease was included as a fixed effect (in a linear model) (Steinbock et al., 2003). Existence of a maternal genetic variation for stillbirth, which is independent of the maternal effect of calving ease, also seems plausible. Steinbock et al. (2003) and Meyer et al. (2001b) found a maternal heritability for stillbirth on 0.014 and 0.022 , respectively, when they included calving ease as a fixed effect in a linear model for stillbirth.

\section{Complex Vertebral Malformation}

The models used in this study were limited to assume that the genetic effects (additive and heterosis) were a sum of infinitely many, small independent gene effects. Complex vertebral malformation, which leads to mortality of fetuses and calves probably caused by an autosomal inherited recessive defect (Agerholm et al., 2001), was not accounted for in the model. Nielsen et al. (2003) showed that 77\% of complex vertebral malformation homozygous fetuses were aborted before $260 \mathrm{~d}$ after insemination. As stillbirth is recorded for calvings $260 \mathrm{~d}$ after insemination, the impact of complex vertebral malformation on the inference of quantitative genetic parameters of stillbirth is believed to be limited.

\section{Choice of Model}

In this study we chose to model direct and maternal effects by fitting a model with correlated random effects of sires and MGS. The S-MGS model can be justified as (artificial) selection operates mainly on bulls in dairy cattle. Theoretically, an animal threshold model would be more appropriate than a S-MGS model, but it has been shown to cause problems, which are related to the extreme category problem (ECP) (Hoeschele and Tier, 1995; Luo et al., 2001). The ECP named by Misztal et al. (1989) can arise in threshold models, when all observations in a subclass for a location parameter are

Table 7. Correlations between posterior samples of effects of breed, heterosis and sex from TM-HET with joint sampling of location parameters.

\begin{tabular}{lllcrr}
\hline & $b_{\text {Het-calf }}$ & $b_{\text {HF-dam }}$ & $b_{\text {Het-dam }}$ & Male & Female \\
\hline$b_{\text {HF-calf }}$ & -0.33 & 0.51 & -0.09 & -0.74 & -0.74 \\
$b_{\text {Het-calf }}$ & & 0.40 & 0.44 & -0.35 & -0.35 \\
$b_{\text {HF-dam }}$ & & & -0.26 & -0.79 & -0.79 \\
$b_{\text {Het-dam }}$ & & & & -0.17 & -0.17 \\
male & & & & 1.00 \\
\hline
\end{tabular}


in one of the categories. In a comparison of all combinations of linear, threshold, animal, and S-MGS models with fixed or random CG effects, Luo et al. (2001) found that the threshold S-MGS model with random CG effects performed best for categorical data when Gibbs sampling was used. In a Bayesian analysis of a sire threshold model using Gibbs sampling, Hoeschele and Tier (1995) detected problems when CG effects were given a flat uniform prior. In this case individual solutions for CG subclasses with ECP tended towards plus or minus infinity. Even though $45 \%$ of subclasses for CG effects had ECP, these problems disappeared when a normal prior with an unknown variance was used for the CG effects. Then the posterior means of all the variance components were in agreement with simulated values. However, this approach has been shown to give biased inference in some extreme cases (Moreno et al., 1997; Rekaya et al., 2000). Moreno et al. (1997) found bias when $80 \%$ of the subclasses for CG effects had ECP, but no bias was detected when $60 \%$ of the subclasses for CG effects had ECP. In the present study, the effects of CG were given a normal prior, and only $11 \%$ of the subclasses for CG effects had ECP. Therefore, no bias was expected to occur.

\section{CONCLUSIONS}

Both direct and maternal genetic variation for stillbirth at first calving was found. The correlation between the direct and maternal effect was close to zero. The genetic parameters were similar whether effect of breed and heterosis were fitted or not and whether twins were included or excluded from the analysis. As stillbirth is a trait with economic importance, direct and maternal effects of stillbirth could be considered in the breeding goal. If not included in the breeding goal, a breeding value evaluation to identify sires with extreme breeding values and to follow the genetic development of the direct and maternal effect is recommended.

\section{REFERENCES}

Agerholm, J. S., C. Bendixen, O. Andersen, and J. Arnbjerg. 2001. Complex vertebral malformation in Holstein calves. J. Vet. Diagn. Invest. 13:283-289.

Albert, J. H., and S. Chip. 1993. Bayesian analysis of binary and polychotomous response data. J. Am. Stat. Assoc. 88:669-679.

Bundgaard, E., and S. Hoej. 2000. Direct access to the cattle database with livestock registration. Annu. Rep. 1999, National Committee on Danish Cattle Husbandry, Aarhus, Denmark.

Danish Cattle Federation, 2002. Principles of Danish cattle breeding. http://www.lr.dk/kvaeg/diverse/principles.pdf. Accessed Feb. 18, 2003.

Dempster, E. R., and I. M. Lerner. 1950. Heritability of threshold characters. Genetics 35:212-236.

Garcia-Cortés, L. A., and D. Sorensen. 1996. On a multivariate implementation of the Gibbs sampler. Genet. Sel. Evol. 28:121-126.
Geman, S., and D. Geman. 1984. Stochastic relaxation, Gibbs distributions, and the Bayesian restoration of images. IEEE Trans. Pattern Anal. Machine Intelligence 6:721-741.

Harbers, A., L. Segeren, and G. de Jong. 2000. Genetic parameters for stillbirth in the Netherlands. Proc. of the 2000 Interbull Meeting in Bled, Slovenia, May 14-15, 2000. Interbull Bull. 25:117122.

Harville, D. A., and R. W. Mee. 1983. A mixed-model procedure for analyzing ordered categorical data. Biometrics 40:393-408.

Hoeschele, I., and D. Gianola. 1989. Bayesian versus maximum quasilikelihood methods for sire evaluation with categorical data. J. Dairy Sci. 72:1569-1577.

Hoeschele, I., and B. Tier. 1995. Estimation of variance components of threshold characters by marginal posterior modes and means via Gibbs sampling. Genet. Sel. Evol. 27:519-540.

Jensen, J., E. A. Mäntysaari, P. Madsen, and R. Thompson. 1997. Residual maximum likelihood estimation of (co)variance components in multivariate mixed linear models using average information. J. Ind. Soc. Agric. Stat. 49:215-236.

Korsgaard, I. R., M. S. Lund, D. Sorensen, D. Gianola, P. Madsen, and J. Jensen. 2003. Multivariate Bayesian analysis of Gaussian, right censored Gaussian, ordered categorical and binary traits using Gibbs sampling. Genet. Sel. Evol. 35:159-183.

Liu, J. S., W. H. Wong, and A. Kong. 1994. Covariance structure of the Gibbs sampler with applications to the comparisons of estimators and augmentation schemes. Biometrika 81:27-40.

Luo, M. F. 1999. Bayesian inference for calving ease and stillbirth in dairy cattle. Ph.D. Diss. Univ. Guelph, Canada.

Luo, M. F., P. J. Boettcher, J. C. M. Dekkers, and L. R. Schaeffer. 1999. Bayesian analysis for estimation of genetic parameters of calving ease and stillbirth for Canadian Holsteins. J. Dairy Sci. 82:1848-1858.

Luo, M. F., P. J. Boettcher, L. R. Schaeffer, and J. C. M. Dekkers. 2001. Bayesian inference for categorical traits with an application to variance component estimation. J. Dairy Sci. 84:694-704.

Madsen, P., and J. Jensen. 2000. DMU: A User's Guide. A package for analysing multivariate mixed models. Version 6 , release 4 . DIAS, Foulum, Denmark.

Meijering, A. 1984. Dystocia and stillbirth in cattle-A review of causes, relations and implications. Livest. Prod. Sci. 11:143-177.

Meyer, C. L., P. J. Berger, K. J. Koehler, J. R. Thompson, and C. G. Sattler. 2001a. Phenotypic trends in incidence of stillbirth for Holsteins in the United States. J. Dairy Sci. 84:515-523.

Meyer, C. L., P. J. Berger, J. R. Thompson, and C. G. Sattler. 2001b. Genetic evaluation of Holstein sires and maternal grandsires in the United States for perinatal survival. J. Dairy Sci. 84:12461254 .

Misztal, I., D. Gianola, and J. L. Foulley. 1989. Computing aspects of a nonlinear method of sire evaluation for categorical data. J. Dairy Sci. 72:1557-1568.

Moreno, C., D. Sorensen, and L. A. García-Cortés, L. Varona, and J. Altarriba. 1997. On biased inferences about variance components in the binary threshold model. Genet. Sel. Evol. 29:145-160.

Nielsen, L. A. H., A. Glacius, A. Fogh, and F. Skjøth. 2002. Dødelighed hos kalve af malkerace. Rep. no. 102. Danish Cattle, Udkærsvej 15, Aarhus, Denmark.

Nielsen, U. S., G. P. Aamand, O. Andersen, C. Bendixen, V. H. Nielsen, and J. S. Agerholm. 2003. Effects of complex vertebral malformation on fertility traits in Holstein cattle. Livest. Prod. Sci. 79:233-238.

Niskanen, J., and J. Juga. 1998. Calving difficulties and calf mortality in Finnish dairy cattle population. Proc. International Workshop on Genetic Improvement of Functional Traits in Cattle; Fertility and Reproduction, Grub, Germany, November, 1997. Interbull Bull. 18:17-20.

Philipsson, J. 1976. Studies on calving difficulty, stillbirth and associated factors in Swedish cattle breeds III. Genetic parameters. Acta Agric. Scand. 26:211-220.

Philipsson, J., J. L. Foulley, J. Lederer, T. Liboriussen, and A. Osinga. 1979. Sire evaluation standards and breeding strategies for limiting dystocia and stillbirth. Report of an EEC/E.A.A.P working group. Livest. Prod. Sci. 6:111-127. 
Rekaya, R., K. A. Weigel, D. Gianola, B. Heringstad, and G. Klemetsdal. 2000. Methods for attenuating bias of variance component estimates in threshold models when herds are small. J. Dairy Sci. 83:(Suppl. 1):56-57. (Abstr.)

Schmitz, F., R. W. Everett, and R. L. Quaas. 1991. Herd-year-season clustering. J. Dairy Sci. 74:629-636.

Sorensen, D., S. Andersen, D. Gianola, and I. Korsgaard. 1995. Bayesian inference in threshold models using Gibbs sampling. Genet. Sel. Evol. 27:229-249.

Sorensen, D., and D. Gianola. 2002. Likelihood, Bayesian, and MCMC Methods in Quantitative Genetics. Springer-Verlag, New York, NY

Steinbock, L., A. Näsholm, B. Berglund, K. Johansson, and J. Philipsson. 2003. Genetic effects on stillbirth and calving difficulty in Swedish Holsteins at first and second calving. J. Dairy Sci. 86:2228-2235.

Van Vleck, L. D. 1972. Estimation of heritability of threshold characters. J. Dairy Sci. 55:218-225.

Van Vleck, L. D. 1985. Including records of daughters of selected bulls in estimation of sire component of variance. J. Dairy Sci. 68:2396-2402.

Weller, J. I., I. Misztal, and D. Gianola. 1988. Genetic analysis of dystocia and calf mortality in Israeli-Holsteins by threshold and linear models. J. Dairy Sci. 71:2491-2501.

Willham, R. L. 1972. The role of maternal effects in animal breeding: III. Biometrical aspects of maternal effects. J. Anim. Sci. 35:1288-1292.

Wright, S. 1934. An analysis of variability in number of digits in an inbred strain of guinea pigs. Genetics 19:506-536. 